\title{
Aerocapture Guidance and Performance at Mars for High-Mass Systems
}

\author{
Carlie H. Zumwalt, Ronald R. Sostaric, and Carlos H. Westhelle \\ NASA Johnson Space Center, Houston, TX, 77058, USA
}

The Mars Entry, Descent, and Landing System Analysis (EDL-SA) project has been tasked with performing systems analysis to identify the optimal technologies required to land a 20-50 MT Exploration-class mission on Mars. It has been shown that it is not possible to safely land these large systems using heritage Mars EDL systems, or analogous Earth or Moon EDL systems. In 2007, NASA conducted a Mars Exploration Architecture Study ${ }^{1}$ which included an in depth review of the science motivations and engineering technology requirements for a human Mars mission campaign. This study resulted in an update to the Mars Design Reference Architecture (DRA 5.0). Among the primary findings and recommendations was the conclusion that landing of large payloads (greater than $1 \mathrm{MT}$ ) on the surface of Mars remains a key architectural challenge. Additionally, research and system studies of fundamental EDL technologies were highly recommended.

The EDL-SA project identified the candidate technologies and assembled them into full capture and EDL sequences so that simulations could be developed to evaluate them. The chosen architectures (shown in Figure 1) combine the various technologies of interest in eight different ways. For aerocapture, only two scenarios were considered. The first is a rigid mid-L/D aeroshell (AS), which is represented in architectures 1, 4, 5 , and 7. This scenario calls for a vehicle that flies at a 55-degree angle of attack, resulting in ballistic coefficient and L/D values of $490 \mathrm{~kg} / \mathrm{m}^{2}$ and 0.43 , respectively. The second is a lifting hypersonic inflatable aerodynamic decelerator (HIAD), which is represented in architectures 2, 6, and 8. This scenario requires the vehicle to fly at a 22.2degree angle of attack, which correlates to an L/D of 0.3 and was sized to provide a ballistic coefficient of 165 $\mathrm{kg} / \mathrm{m}^{2}$. Architecture 3 is an all-propulsive sequence, and is not considered in this study.

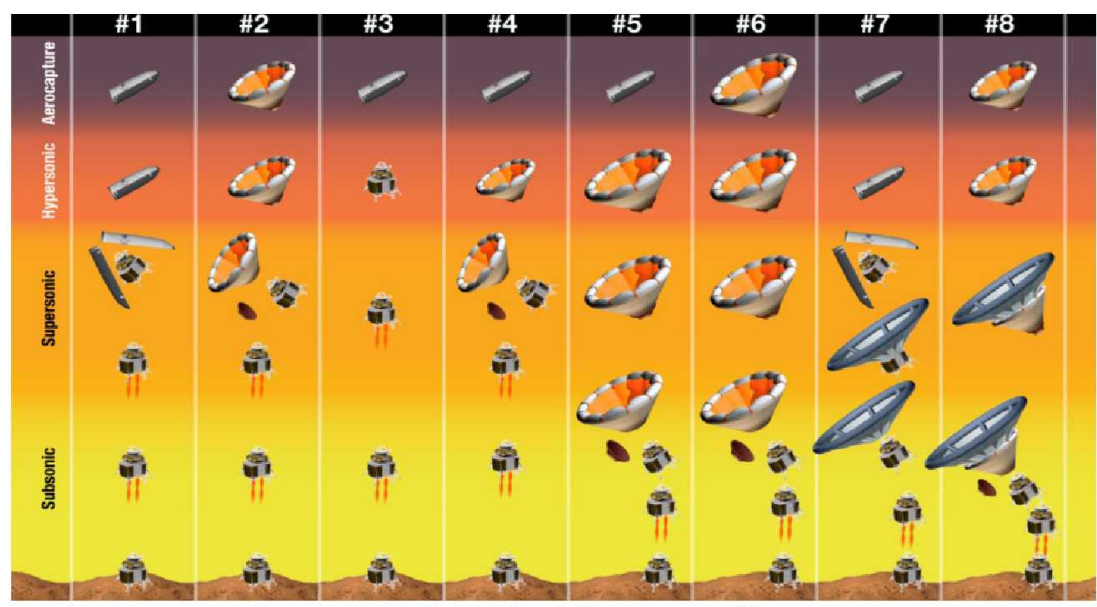

Figure 1 EDL-SA Architectures Diagram

For both configurations, Monte Carlo performance and sensitivity analysis were completed using the POST2 simulation at LaRC. This simulation uses the Hybrid Predictor Corrector Aerocapture Scheme (HYPAS) guidance algorithm to simulate the aerocapture phase. Aerocapture is used to slow the vehicle down from a hyperbolic approach trajectory to an elliptical orbit around the desired landing body, prior to EDL. Figure 2 shows the aerocapture phases, which are applicable to both scenarios. HYPAS is an analytical predictor-corrector algorithm that was developed and selected for the Aeroassist Fight Experiment, an aerocapture demonstrator mission that was canceled before launch. HYPAS targets a lifting vehicle through the atmosphere to a desired exit orbit apoapsis and inclination by using an analytically derived control algorithm. This algorithm uses deceleration due to drag and altitude rate error feedback to determine the bank angle magnitude, and the inclination error to determine bank direction. It has been used in numerous human and robotic exploration mission studies over the last 10 years for Earth and Mars, and has been proven to be robust to a wide variety of $L / D, m / C_{D} S$ (ballistic coefficients), atmospheres, entry conditions, and target orbits. 


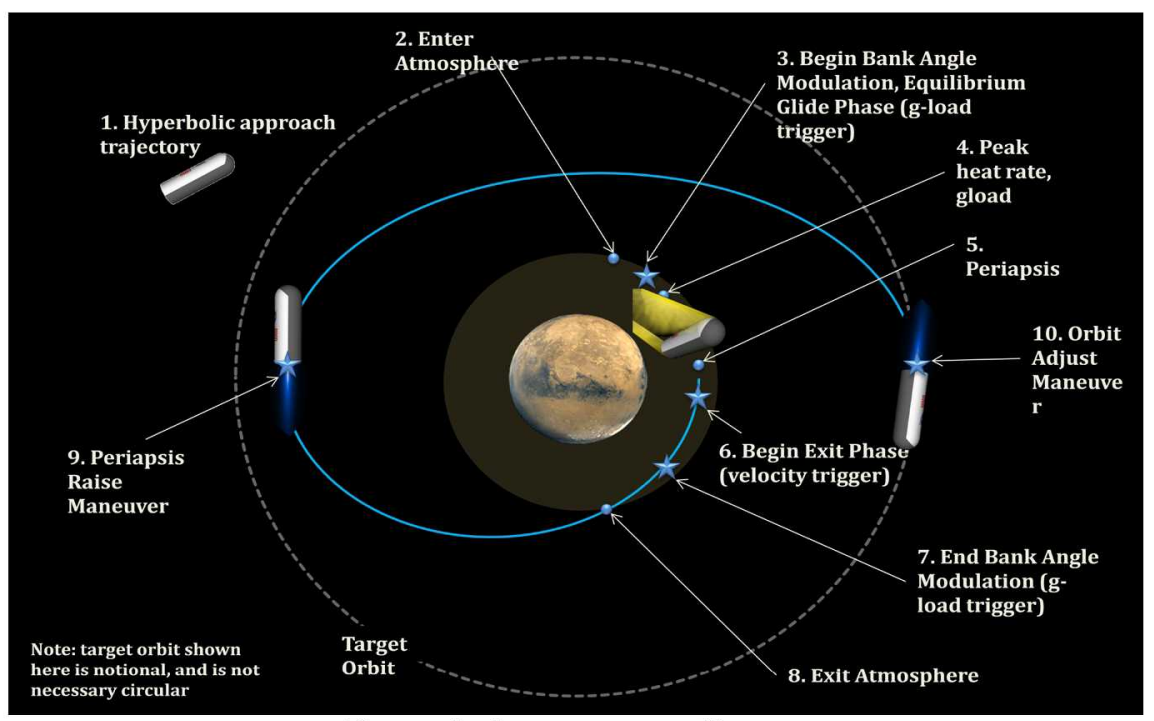

Figure 2 Aerocapture Phases

Reference trajectories were developed for both the rigid and inflatable aeroshell designs, using both a 1 sol and $500 \mathrm{~km}$ target orbit, and these are used for the basis of all analysis. For each of the 4 trajectories, 2000 case Monte Carlo sets were run, and the HYPAS gains were optimized for $\Delta \mathrm{V}$ performance within a set (i.e. 4 sets of gains were found, 1 set for each trajectory). The set of applied dispersions is described in [2].

The first task was to study the performance in the nominals of each of the four specific vehicle designs described previously. Results showed good performance across the entire set of cases. The budgeted $\Delta V$ for the periapse raise maneuver and the orbit adjust maneuver (phases 9 and 10 of Figure 2) was $150 \mathrm{~m} / \mathrm{s}$. The mean $\Delta V$ for the rigid, $1 \mathrm{sol}$ case was $16.94 \mathrm{~m} / \mathrm{s}$ with a standard deviation of $3.71 \mathrm{~m} / \mathrm{s}$, and for the inflatable, had a mean of 16.05 $\mathrm{m} / \mathrm{s}$ and a standard deviation of $3.49 \mathrm{~m} / \mathrm{s}$. It was concluded that the lower L/D of the inflatable does not affect the $\Delta V$ performance for the regime studied, because the guidance is robust enough to handle the applied dispersions. Additionally, a large number of sensitivity cases were also completed in order to evaluate parameter changes outside of those defined in the Monte Carlo variations. These include mass, the aforementioned target orbit, and three different atmosphere model inputs (solar longitude, dust tau, and time of day). An examination of the results for the nominal cases showed no significant variation in $\Delta V$ performance across the entire set of these runs, however the difference in $\Delta \mathrm{V}$ required for the post-aerocapture cleanup maneuvers, roughly $100 \mathrm{~m} / \mathrm{s}$, shows that the choice of target apoapsis (from 33,793 km to $500 \mathrm{~km}$ ) proves to have a significant effect on $\Delta \mathrm{V}$. In combination with the demonstrated robustness using HYPAS to the Monte Carlo dispersions and sensitivities, the aerocapture results show excellent performance for the selected architectures and configurations.

Given the excellent performance demonstrated by HYPAS in task one, task two was created to determine how low in L/D and Ballistic coefficient the vehicle could fly before it began to exhibit poor performance. For this, L/D were studied in the range of 0.1 to 0.3 , and Ballistic coefficients in the range of 25 to $50 \mathrm{~kg} / \mathrm{m}^{2}$. Sixteen L/D-Bc combinations were created, and 1000 case Monte Carlo runs were completed for each. Results show that performance is highly sensitive to $L / D$, and that poor performance is observed for any $L / D$ lower than 0.25 . Below and L/D of 0.25 , the amount of corridor margin, or the difference between the flight path angle flown and the flight path angle needed to cause skip-out, drops below 1 degree and the vehicle begins to miss its apoapse target by as much as $70,000 \mathrm{~km}$. It should be noted, however, that in no instance were any cases lost to skip-out or surface impact, rather the $\Delta V$ required to clean up the orbit far exceeds the $150 \mathrm{~m} / \mathrm{s}$ budgeted. Therefore, for each of the Ballistic coefficient values studied, the lowest L/D available for good performance is 0.25 .

\section{References}

1. B.G. Drake (ed.), Human Exploration of Mars Design Reference Architecture 5.0, NASA-SP-2009-566, July 2009

2. Report of the Design Reference Missions, Ground Rules and Assumptions, and Figures of Merit, EDLSA-001 Rev A, Sept. 1, 2009 